\title{
Masaai warrior shield: The life time of a three body system
}

\author{
Harry J. Lehto ${ }^{1,2}$, Pekka Heinämäki ${ }^{1}$ Arthur D. Chernin ${ }^{1,3}$ and Mauri J. \\ Valtonen ${ }^{1,2}$ \\ ${ }^{1}$ Tuorla Observatory, Turku University, 21500 Piikkiö, Finland \\ ${ }^{2}$ Department of Physical Sciences, 20014 Turku University, Finland \\ ${ }^{3}$ Sternberg Astron. Institute, Moscow University, 119899 Moscow, \\ Russia
}

\begin{abstract}
We have investigated the behavior of a general Newtonian three body system using the simplest initial conditions. We calculated the evolution of about a million bound three body systems. Within our resolution these cover all the possible initial states. All these systems eventually decay with one body being ejected from the residual binary system. The overall pattern representing the decay time scales projected on a homology map is dominated by resonances. In general appearance it reminds us of a traditional Masaai warrior shield.
\end{abstract}

\section{Introduction - our previous studies}

We have previously determined (Heinämäki et al. 1998) that chaotic behavior in triple systems can be characterized by a transient strange attractor with $D \approx 2.1$. (at least with a certain set of initial conditions).

We have also investigated the behavior of very slightly deviating initial conditions (homology drop) and calculated parameters analogous to the Lyapunov exponent and Kolmogorov-Sinai entropy (Heinämäki et al. 1999).

\section{Homology triangle}

A three body configuration can be specified in the following way (Agekian and Anosova, 1967): Scale, rotate and translate the initial configuration in such a way that the body adjacent to the two longest sides is located at $X_{1}=(0,0)$. Let the body that is between the longest and the shortest side be located at $X_{2}=(1,0)$. Now the location of the third body $X_{3}$ is in a homology triangle, and uniquely defines the geometry of the system.1b).

\section{Simulations}

Initially our three body configuration is at rest with no net angular momentum. The evolution of the system is determined only by the mutual gravitational fields of the equal mass objects. We calculated the evolution of all possible 
orbits represented by 200,000 orbits (fig. 1). We further zoomed into certain areas of the parameter space and calculated a further $\sim 250,000$ orbits at each level.

We followed every triple configuration until one of the components was ejected. Ejection was defined to have taken place if one of the bodies had a non-negative energy and a distance of $r \geq 1$ to the two remaining bodies. We expanded the homology triangle to all four quarters for future studies of unequally spaced masses. In the present simulations the lifetimes of the complete system can be described by the "warrior shield".

\section{Masaai warrior shield}

The warrior shield (Fig. 1) represents the decay time scales of all possible configurations of a three body system by a color ladder from scales of black and white. The solid gray strips represent the systems that are short lived, $\leq 1$ crossing time scale, light gray arcs survive up to 2 crossing time scales, and the darkest ones survive over 13 crossing times. The structure that emerges is quite complicated. On largest scales we can identify the following features:

- a vertical solid gray strip across the center of the map. This is the 1:1 resonance.

- a set of unstable solid gray srips. These represent the odd-digit orbital resonances $(1: 3,1: 5,1: 7 \mathrm{etc})$. Note that even-digit resonances are completely missing, because the phase in the first encounter is not favorable for rapid disintegration (Saslaw, Valtonen, Aarseth, 1974).

- Systems breaking up after second interaction appear as long arcs or loops. These are created by an odd-number resonance after the first encounter, except for the system of arcs ending at the main 1:1 resonance band, where also even number resonances are present.

- All the major resonances appear to avoid the sea of tranquillity located around $(x, y)=(0.7,0.5)$.

- Locally the boundaries of the instability regions are sharp from the inside and possess ever denser stripes and loops on the outside, appearing selfsimilar.

\section{Life times of closeby orbits}

If $\left|t_{\text {med }}-t_{o}\right| \leq 0.5$, we considered the point to have a locally homogeneous neighborhood. Otherwise it was considered to have high sensitivity to initial conditions, a characteristic of chaos. Of the 21,699 cases compared in this manner 6,212 , or $p_{\epsilon}=28.6 \%$ were locally homogeneous.

The remaining $71.4 \%$ positions turned out to be highly sensitive to initial conditions at this resolution, implying that on the scale of $\epsilon=1.25 \times 10^{-3}$ the fractal area covers about $71.4 \%$ of all the systems. This means that one will be able to "predict" the disintegration time scale with a success probability of 
$28.6 \%$, if one knows the disintegration timescales of the neighbours, located at a distance of $\epsilon$. By studying this predictability behavior at different scales we find that the Hausdorff dimension of our image is $D_{H}=1.5$.

\section{Regions of stability}

We find two different sets of regions of high stability. The first is a set of isolated deep trenches on large scales at about $(0.70,0.64)$ and $(0.65,0.15)$. The second set, visible on small scales, are configurations just outside some instability regions e.g. around the upper branch of $(1: 7)$ at $(0.90,0.23)$ or below the area at $(0.75,0.60)$. All these areas have orbits with very long life-times.

\section{Discussion}

We have calculated the evolution of a complete set of orbits with the initial conditions specified earlier. The unit time scale of our $(G=1)$ system is

$$
t=\frac{1}{2 \pi}\left(\frac{R}{1 \mathrm{AU}}\right)^{3 / 2} \sqrt{\frac{M_{\odot}}{M}} \text { years, }
$$

where $R$ is the longest separation of the triangle and $M$ is the mass of the objects. (Point-like) galaxies at distances of $\sim 0.5 \mathrm{Mpc}$, and with masses $M \sim 50 \times 10^{9} M_{\odot}$ have a typical time scale of $10^{10}$ years, so many triple systems have not disintegrated, or are just about to disintegrate. One should note though that timescales of systems with net angular momentum or non-zero velocity are likely to be somewhat longer based on previous work (Saslaw, Valtonen and Aarseth, 1974).

\section{References}

Agekian, T. A. and Anosova, J. P. 1967, Sov. Astron., 44, 162

Heinämäki, P., Lehto, H. J, Chernin A. C. and Valtonen, M. J. 1998, MNRAS, 298, 790

Heinämäki, P., Lehto, H. J., Chernin A. C. and Valtonen, M. J., 1999, MNRAS, in press

Mikkola, S. and Aarseth, S. 1996, Celestial Mechanics and Dynamical Astronomy, 64, 197

Saslaw, W. C., Valtonen. M. J, Aarseth, S. J. 1974, ApJ, 190, 253 


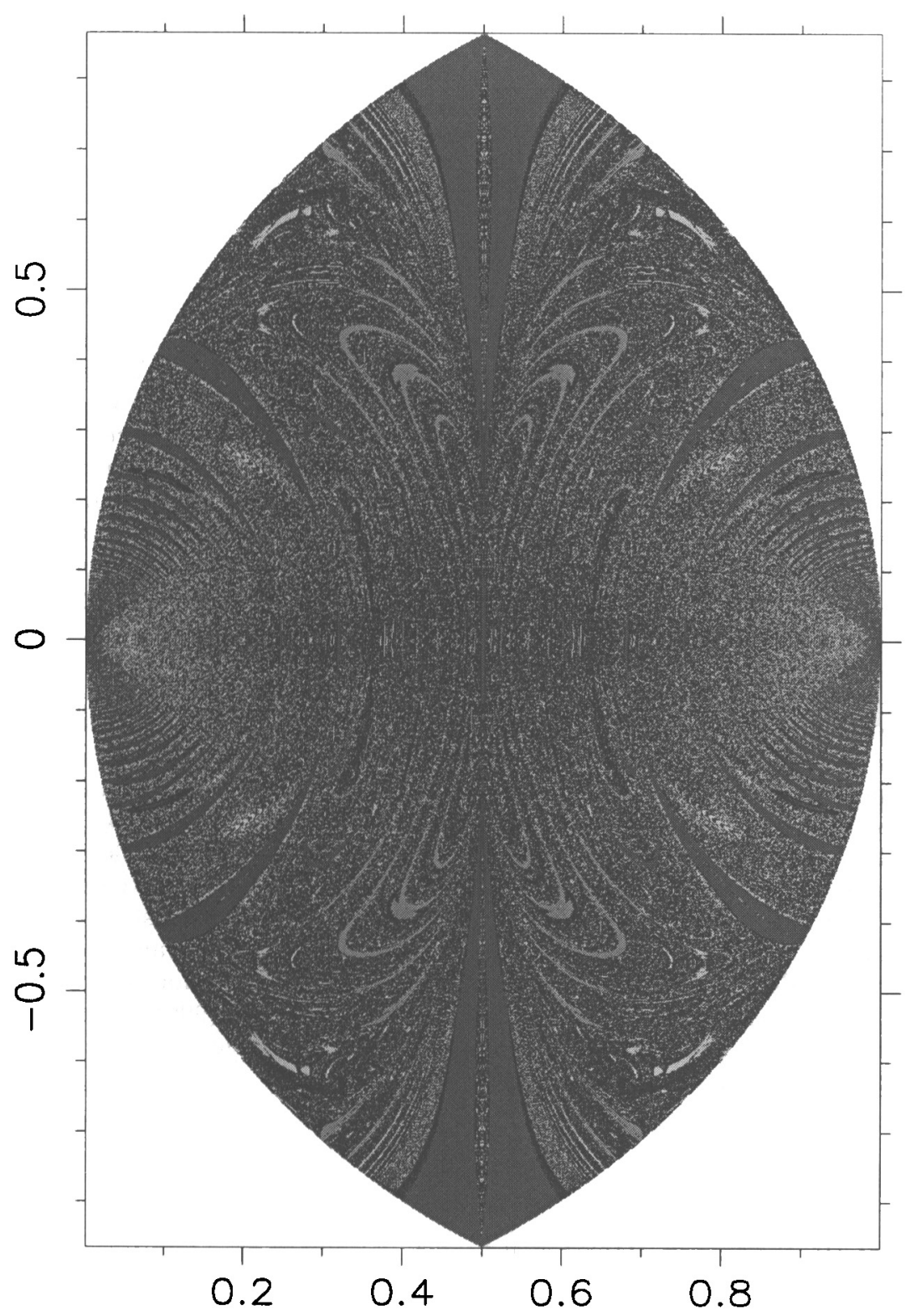

Figure 1. Expanded homology triangle shows decay time scales of all possible configurations of a three body system 